\title{
Helicobacter pylori with stronger intensity of CagA phosphorylation lead to an increased risk of gastric intestinal metaplasia and cancer
}

Chiao-Hsiung Chuang ${ }^{1}$, Hsiao-Bai Yang ${ }^{2}$, Shew-Meei Sheu ${ }^{3}$, Kuei-Hsiang Hung ${ }^{3}$, Jiunn-Jong Wu ${ }^{3}$, Hsiu-Chi Cheng ${ }^{1,4}$ , Wei-Lun Chang ${ }^{1,4}$ and Bor-Shyang Sheu ${ }^{1,3,4^{*}}$

\begin{abstract}
Background: Nearly all Taiwanese H. pylori stains are cagA-genopositive and encode CagA protein. In this study, we evaluated whether different intensity of tyrosine phosphorylated-CagA ( $p-C a g A$ ) had an impact on the clinical diseases and histological outcomes in this area.

Results: We enrolled 469 dyspeptic patients and prospectively obtained the gastric biopsy specimens and the $H$. pylori isolates. These patients were categorized according to the clinical diseases, such as duodenal ulcer, gastric ulcer, gastric cancer, and gastritis with or without intestinal metaplasia. Their gastric specimens were reviewed by the updated Sydney's system. Furthermore, a total of 146 patients were randomly selected from each clinical category for evaluation of their isolates' p-CagA intensity by in vitro AGS cells co-culture. The p-CagA was sparse in $30(20.5 \%)$, weak in 59 (40.5\%), and strong in 57 (39\%) isolates. The isolates from the patients of gastric cancer or gastritis with intestinal metaplasia had stronger $\mathrm{p}$-CagA intensity than those of gastritis without intestinal metaplasia $(p \leq 0.002)$. Moreover, the patients infected with isolates with strong or weak p-CagA intensity had a higher risk of gastric intestinal metaplasia $(p<0.05$, odds ratio 3.09 15.26) than those infected with sparse p-CagA isolates.
\end{abstract}

Conclusions: Infection with H. pylori stains with stronger p-CagA intensity may lead to an increased risk of gastric intestinal metaplasia and cancer.

Keywords: H. pylori cagA, CagA phosphorylation, intestinal metaplasia, gastric cancer

\section{Background}

The cagA gene encoded CagA protein is a well-known virulent factor of Helicobacter pylori, which is associated with an increased risk of peptic ulcer or even gastric cancer [1-4]. The CagA protein can be tyrosine phosphorylated in the gastric epithelial cells via the type IV secretion system translocation [5]. The phosphorylated-CagA (p-CagA) mediates interleukin-8 secretion, enhances gastric inflammation, and clinical diseases [5-8]. As shown in the Mongolian gerbil models, H. pylori isolates with functional type IV secretion system could induce more CagA phosphorylation and

\footnotetext{
* Correspondence: sheubs@mail.ncku.edu.tw

'Department of Internal Medicine, Medical College, National Cheng Kung University, Sheng-Li Road, Tainan, Taiwan

Full list of author information is available at the end of the article
}

severer gastric inflammation and intestinal metaplasia (IM) $[9,10]$. However, there is no adequate clinical evidence in a setting to support the relationship between CagA phosphorylation intensity and the risk of gastric carcinogenesis.

In the western countries, about $70 \%$ or less of clinical $H$. pylori strains are cagA-genopositive [11,12]. In contrast, in the eastern countries, such as in Taiwan, there is a nearly $100 \%$ prevalence of cagA-vacA-babA2 triplepositive $H$. pylori strains [13-15]. Moreover, most strains in East-Asia, and also Taiwan, encoded CagA contain EPIYA-ABD motif [16-18]. Our previous data supported $100 \%$ positive of some genes which are encoded from cag pathogenicity island (PAI), such as cagC, cagE, cagF, $\operatorname{cagN}$, and $\operatorname{cagT}$ [19]. Accordingly, because of the universal presence of genes in cag-PAI in Taiwan, this 
region should be suitable to answer whether different pCagA intensity are related to different clinicopathologic outcomes of $H$. pylori infections. The study is highly original to illustrate the $\mathrm{p}$-CagA intensity could be diverse

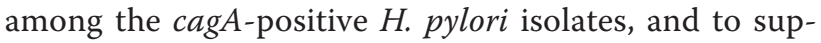
port $H$. pylori with stronger p-CagA intensity can increase the risk of gastric carcinogenesis.

\section{Methods}

\section{Patients and study design}

Patients with recurrent dyspepsia symptoms, who received upper gastrointestinal endoscopy, were consecutively enrolled, once they were proven to have a $H$. pylori infection defined by a positive result of culture. None of them had a previous history of anti-H. pylori therapy. For each patient, the gastric biopsies were obtained during the endoscopy for $H$. pylori culture and histological analysis. This study were approved by 'Human Experiment and Ethics Committee of National Cheng Kung University Hospital' (ER-97-245) and all the patients signed the informed consents before enrollment.

A total of 469 patients (264 women and 205 men; mean age 48.1 years) were enrolled, including 26 with gastric cancer, 64 with gastric ulcer, 131 with duodenal ulcer, 209 with gastritis \& without IM and 39 with gastritis \& IM. From each category, 32 isolates were randomly sampled (the cancer group had just 26 isolates and all were selected). A total of 154 isolates were sampled, but 8 stored strains could not be successfully subcultured after refrigeration. Accordingly, 146 strains were finally obtained from patients with duodenal ulcer $(n=31)$, gastric ulcer $(n=32)$, gastric cancer $(n=24)$, gastritis with IM $(\mathrm{n}=28)$, and gastritis without IM ( $\mathrm{n}=$ 31). These $146 \mathrm{H}$. pylori isolates were analyzed for the cagA-genotype by polymerase chain reaction and for the intensity of p-CagA by in vitro co-culture with AGS cells (a human gastric adenocarcinoma epithelial cell line); further the p-CagA intensity was defined as strong, weak, or sparse. Besides, in each patient, their gastric biopsies taken from both antrum and corpus for histology were reviewed by the updated Sydney's system.

\section{Histological analysis of the gastric specimens}

Each gastric sample was stained with haematoxylin and eosin as well as with modified Giemsa stains to analyze for $H$. pylori density (HPD, range $0-5$ ) and $H$. pylorirelated histology by the updated Sydney's system. The histological parameters included acute inflammation score (AIS, range 0-3; 0: none, 1: mild, 2:moderate, 3: severe), chronic inflammation score (CIS, range $1-3 ; 1$ : mild, 2: moderate, 3: severe), mucosal atrophy, and IM as applied in our previous studies [20,21]. For each patient, the presence of atrophy or IM was defined as a positive histological finding in any specimen from the antrum or corpus. In each patient, the total HPD, AIS, and CIS were the sum of each score of the gastric specimens from antrum and corpus, and thus ranged from 0 $10,0-6$, and 2-6, respectively. Based on the sum of HPD, the patients were categorized as loose (score $\leq 5$ ), moderate (score within 6-8), and dense (score $\geq 9$ ) H. pylori colonization, respectively. For the sum of AIS, mild, moderate, and severe acute inflammations were defined with scores $\leq 1,2-3$, or $\geq 4$, respectively. Based on the sum of CIS, mild, moderate, and severe chronic inflammations were defined with scores $\leq 3,4-5$, or 6 , respectively.

Based on the specimens collected from both the antrum and corpus within the same patient, the topographical distribution of chronic gastritis was defined as follows: 1) very limited chronic gastritis, if the CIS scored was 1 for both antrum and corpus; 2) antrumpredominant gastritis, if the CIS score of the antrum was higher than the score of the corpus; and 3) corpuspredominant gastritis, if the corpus CIS was equal to or higher than that of the antrum [21].

\section{Analysis of cagA genotype and type IV secretion system function of $\mathrm{H}$. pylori}

All $H$. pylori isolates were re-sorted by culture as applied in our previous publications $[14,15]$. The genomic DNA of these collected isolates was then extracted for polymerase chain reaction to verify the cagA-genotype by primers used in our published article [19]. To analyze the p-CagA intensity of each strain, $H$. pylori strains $\left(2 \times 10^{8}\right.$ cells $)$ were suspended in $0.5 \mathrm{~mL}$ of phosphate-buffered saline (PBS) and were co-cultured with $2 \times 10^{6}$ AGS cells at a multiplicity of infection (MOI) of 100 for 5 hours. Afterward, the culture medium was removed and the AGS cells were lysed after five times washing with PBS. The AGS lysates were applied to SDS-PAGE gel electorphoresis and transferred to membranes for western blots analysis. A phosphorylated tyrosine antibody and anti-actin antibody (Santa Cruz Biotechnology, Inc, Santa Cruz, CA) were used to detect the $\mathrm{p}$-CagA and $\beta$-actin proteins. A clinical H. pylori strain (Hp830) which had a strong p-CagA band in the western blots was used as reference. In each western blots procedure, 7-9 clinical strains and the reference strain were analyzed in the same run. The relative immunoblot density of the $\mathrm{p}$-CagA and $\beta$-actin proteins were quantitated by scanning the images on a gel analysis system (BioSpectrum AC Imaging System, Vision Work LS software, Upland, CA) for each strain and defined as [p-CagA] and [Bactin]. The amount of pCagA and $\beta$-actin proteins of the reference strain in the same run were also semi-quantified as reference and defined as [p-CagA-ref] and [Bactin-ref]. The p-CagA 


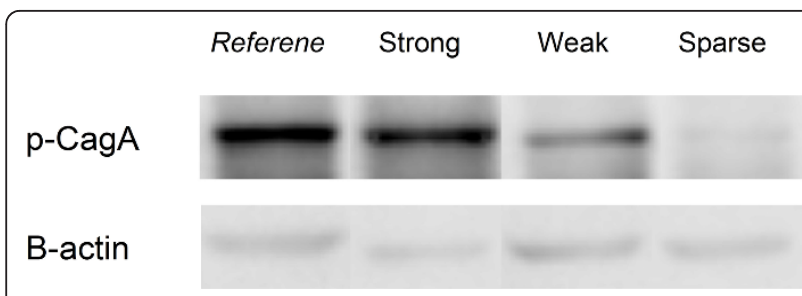

Figure 1 The $p$-CagA and $\beta$-actin immunoblot gel imaging of the reference strains (Hp830) and the representative strain in each subgroup.

intensity of each strain was calculated by the formula: $p$ CagA value $=([p-$ CagA $] /[$ Bactin $]) /([p-$ CagA-ref $] /[$ Bactin ref]). Strains with a p-CagA value $<0.2,0.2-0.8$, and $>0.8$ were defined as sparse, weak, and strong p-CagA intensity. The immunoblot gel imaging of the representative strain in each subgroup and the reference strain (Hp830) were showed in Figure 1.

\section{Statistical analysis}

SPSS software version 12.0 for Windows (SPSS Inc., Chicago, IL) was used for the statistical analysis. The differences in the p-CagA intensity among the subgroups of patients were analyzed by Pearson chi-square test. The odds ratio on the risk of IM and corpus-predominant gastritis between the different subgroups were analyzed by the logistical regression. All tests were twotailed, and a p value less than 0.05 were considered significant.

\section{Results}

\section{H. pylori isolates with diverse $\mathrm{p}$-CagA intensity}

From the 469 patients, we sampled 146 strains for the analysis of the p-CagA intensity. The clinical characteristics of these patients were shown in Table 1. In each sampled group, age and gender were matched between the sampled patients and the entire group of patients ( $\mathrm{p}$ = NS). All of the 146 enrolled H. pylori isolates were

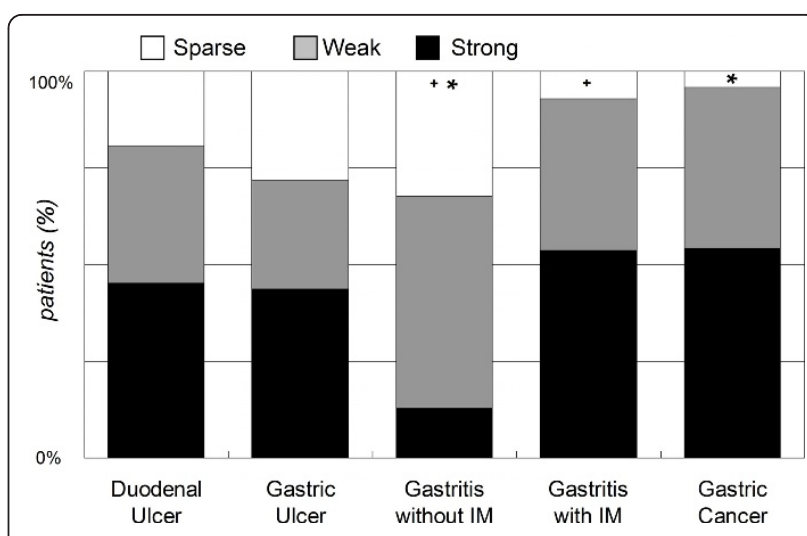

Figure 2 The $p$-CagA intensity of the strains isolated from patients with different clinical categories. The strains isolated from patients of gastric cancer or gastritis with intestinal metaplasia had stronger $\mathrm{p}$-CagA intensity than those from gastritis without intestinal metaplasia patients $\left({ }^{*} p=0.001,{ }^{+} p=0.002\right.$; Pearson chisquare test). IM = intestinal metaplasia.

cagA-genopositive and the p-CagA intensity was sparse in $30(20.5 \%)$, weak in $59(40.5 \%)$, and strong in 57 (39\%) isolates.

\section{Stronger p-CagA intensity may lead to intestinal metaplasia \& gastric cancer}

In Figure 2, the $H$. pylori strains of gastric cancer or gastritis with IM patients had stronger p-CagA intensity than those of gastritis without IM $(54.2 \%$ \& $53.6 \%$ vs. $12.9 \%, p \leq 0.002)$. There was also a trend that the $H$. pylori isolates from cancer or IM patients had relatively stronger p-CagA intensity then the subgroups of gastric and duodenal ulcer, but the difference was not significant. Moreover, the p-CagA intensity was not different among the subgroups of gastric ulcer, duodenal ulcer, and gastritis without IM. In Figure 3, the patients were separated according to having cancer risk or not. The isolates from the patients with cancer or IM had stronger $\mathrm{p}$-CagA intensity than those from non-cancer/IM

Table 1 The clinical characteristics between all patients with isolated $H$. pylori strains and the selected patients for analysis of the p-CagA intensity of the strains

\begin{tabular}{|c|c|c|c|}
\hline & Patients with $H$. pylori cultures $(n=469)$ & Selected patients for $p$-CagA analysis $(n=146)$ & $p$ value* \\
\hline Age (year [mean $\pm \mathrm{SD}]$ ) & $48.1 \pm 14.2$ & $50.4 \pm 16.3$ & NS \\
\hline Gender (F/M) & $264 / 205$ & 73/73 & NS \\
\hline \multicolumn{4}{|l|}{ Endoscopic diagnosis (year; $n(F / M)$ ) } \\
\hline \multicolumn{4}{|l|}{ Gastritis } \\
\hline - without intestinal metaplasia & 44.3; $209(137 / 72)$ & $41.2 ; 31(18 / 13)$ & NS \\
\hline - with intestinal metaplasia & $54.5 ; 39(29 / 10)$ & $57.0 ; 28(22 / 6)$ & NS \\
\hline Duodenal ulcer & $48.0 ; 131(68 / 63)$ & $46.6 ; 31(14 / 17)$ & NS \\
\hline Gastric ulcer & $51.3 ; 64(17 / 47)$ & $49.5 ; 32(7 / 25)$ & NS \\
\hline Gastric cancer & $60.4 ; 26(13 / 13)$ & $60.6 ; 24(12 / 12)$ & NS \\
\hline
\end{tabular}

* Either the age or the gender was matched between the 146 selected patients and the entire patients in each sampled groups (Pearson chi-square test for gender \& Student's $t$ test for age analysis). 


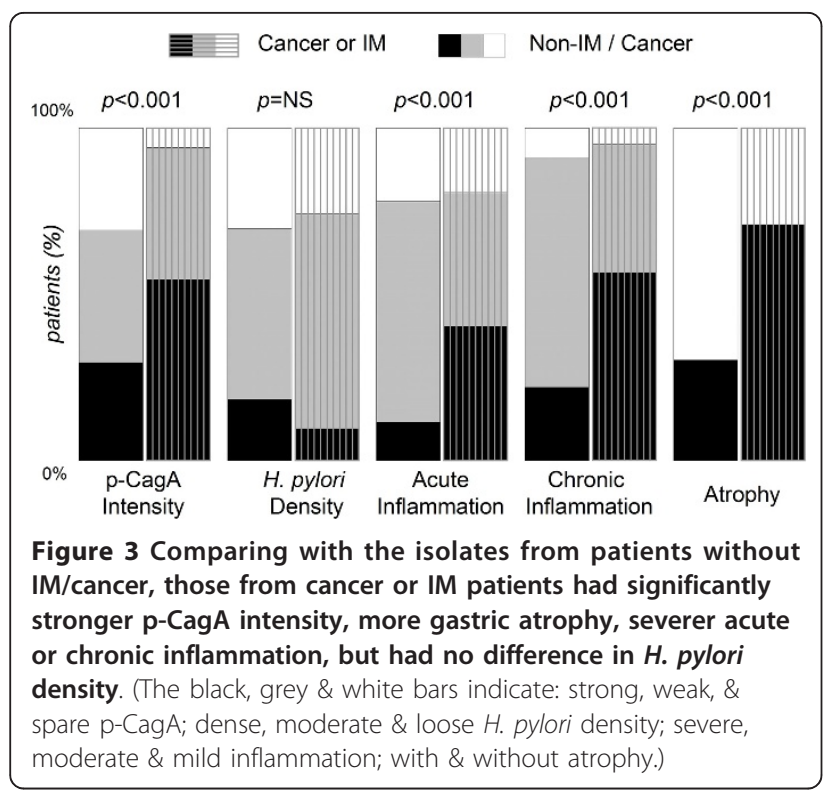

patients $(\mathrm{p}<0.001)$. Furthermore, the patients with cancer risk had higher gastric inflammation or atrophy $(\mathrm{p}<$ 0.001 ).

The impacts of p-CagA intensity on gastric IM were analyzed in the non-cancer patients. Twenty-four out of the 47 patients (51.1\%) infected with strong p-CagA strains had gastric IM. In contrast, for those with weak and sparse p-CagA, 35.4\% (17 out of 48 ) and 11.1\% (3 out of 27) patients had gastric IM. Accordingly, the patients infected with stronger p-CagA strains had higher prevalence of IM in the gastric specimens ( $p=$ 0.003). Moreover, the odds ratios of age, gender and $p$ -

Table 2 The impacts of the p-CagA intensity of $\boldsymbol{H}$. pylori on the gastric intestinal metaplasia in the 122 selected non-cancer patients by stratified analysis and logistical regression

\begin{tabular}{cl}
\hline & Odd ratio $(95 \% \mathrm{Cl})$ \\
\hline Crude: Age $<50$ years & 1 \\
$<50$ years & $8.14(3.49 \sim 18.98)$ \\
Gender - Male & 1 \\
- Female & $2.36(1.12 \sim 5.11)$ \\
p-CagA - Sparse & 1 \\
- Weak & $4.38(1.15 \sim 16.72)$ \\
- Strong & $8.34(2.21 \sim 31.55)$ \\
Age and gender adjusted & 1 \\
Sparse p-CagA & $3.67(0.93 \sim 14.37)$ \\
Weak p-CagA & $8.44(2.08 \sim 34.12)$ \\
Strong p-CagA & \\
Age, gender and disease adjusted & 1 \\
Sparse p-CagA & $3.93(0.92 \sim 16.94)$ \\
Weak p-CagA & $10.45(2.25 \sim 48.48)$ \\
Strong p-CagA &
\end{tabular}

CagA intensity on the gastric IM were showed in Table 2. As compared to those infected with strains with sparse p-CagA intensity, the crude odds ratio to have IM was 4.38 for those with weak p-CagA intensity, and increased to 8.34 for those with strong p-CagA intensity. Based on the logistic regression analysis to adjust the age, gender, and clinical diagnoses, the odds ratios to have IM were 3.93 for the patients infected with weak p-CagA intensity isolates and 10.45 for those with strong p-CagA intensity.

\section{Correlation between H. pylori p-CagA intensity and gastric histological features}

In Figure 4, this study also analyzed whether there were an association between the p-CagA intensity and the severity of gastric inflammation in histology. The patients infected with $H$. pylori isolates with stronger pCagA intensity may have more severe acute inflammation $(p=0.04)$ and also chronic inflammation $(p=$ 0.002). Nevertheless, the p-CagA intensity of $H$. pylori isolates was not associated with the HPD or gastric atrophy $(p>0.05)$.

In Figure 5, a higher proportion of patients infected with a strain with strong p-CagA intensity had corpuspredominant gastritis (59.6\%), as compared to those infected with strains with weak $(40 \%)$ or sparse $(25.9 \%)$ $\mathrm{p}$-CagA intensity $(p=0.001)$. The adjusted odds ratio for age, gender, and clinical diagnoses by logistic regression was 3.15 (1.07 9.31) for patients infected with $H$. pylori with strong p-CagA intensity and 1.49 (0.51 4.35) for those infected with strains with weak p-CagA intensity, as compared with those with sparse p-CagA intensity.

\section{Discussion}

This study shows the clinical impacts of H. pylori pCagA intensity on the risk of gastric carcinogenesis. In Taiwan, the H. pylori isolates have universal presence of genes in cag-PAI and expression of CagA [13-16]. On the basis of the semi-quantitative analysis of the translocated p-CagA bands in the western blots, the strains in this study have diverse intensity of p-CagA. To further evaluate the clinical impact of the diverse p-CagA intensity on the clinical outcome, we selected a clinical strain with marked p-CagA to serve as reference index to subgroup the 146 collected strains according to their pCagA intensity into strong, weak, or sparse. Based on this categorization, this study showed that H. pylori isolates with stronger p-CagA were correlated to more severe gastric inflammation and an increased risk of gastric IM and cancer.

The possible factors to affect CagA phosphrylation include the cagA genotype, type IV secretion system, the CagA EPIYA-repeat motif of the strain, and the 


\section{H. pylori Density}

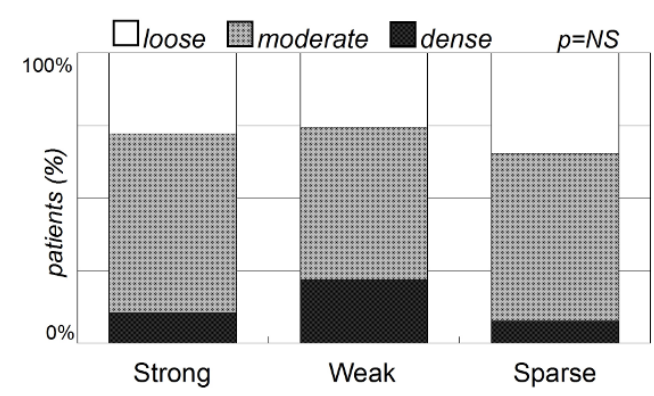

Atrophy

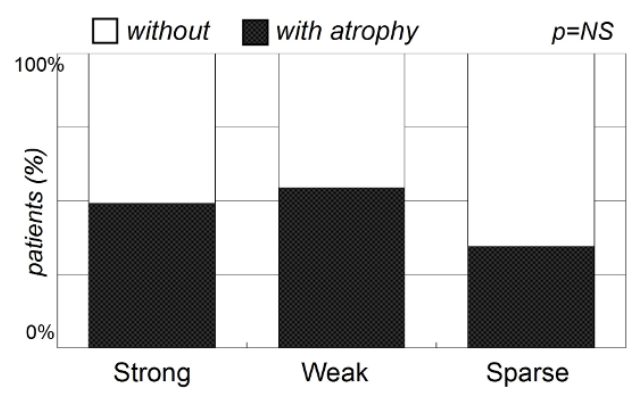

Acute Inflammation

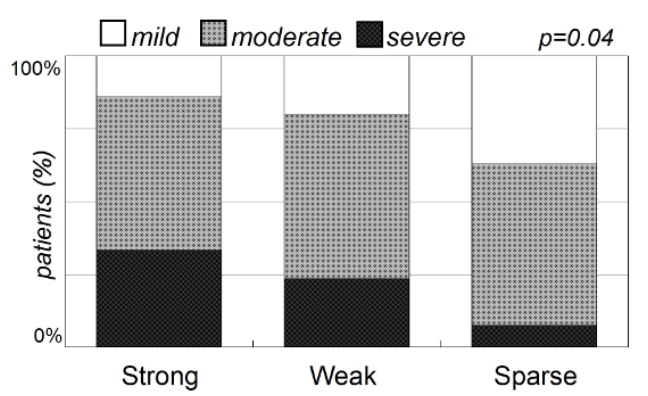

Chronic Inflammation

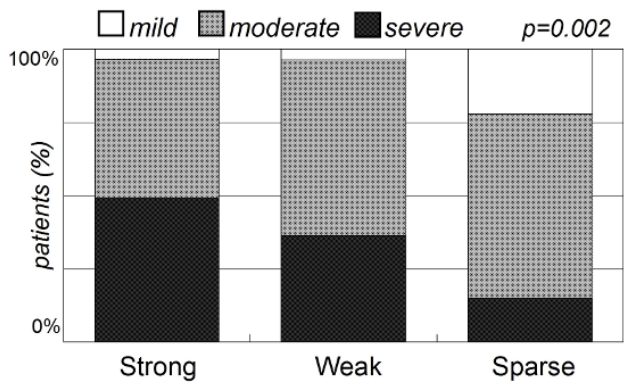

Figure 4 The $H$. pylori density, inflammation and atrophy by gastric histology among the 146 patients infected with $H$. pylori isolates with different $\mathbf{p}$-CagA intensity. The isolates with stronger $\mathrm{p}$-CagA intensity were significantly associated with more severe acute inflammation $(p=0.01)$ and chronic inflammation $(p=0.005)$ but not with H. pylori density or gastric atrophy $(p=N S)$ (Pearson chi-square test).

adhesion phenotype of the epithelial cell [22-27]. Animal studies have shown that mutant strains of CagA, CagE, or CagY could reduce the gastric inflammation after infection [10,28]. Moreover, the CagA EPIYA polymorphism has also a causal role in clinical outcome $[18,29]$. These data support that these factors are all important in the H. pylori related gastric inflammation

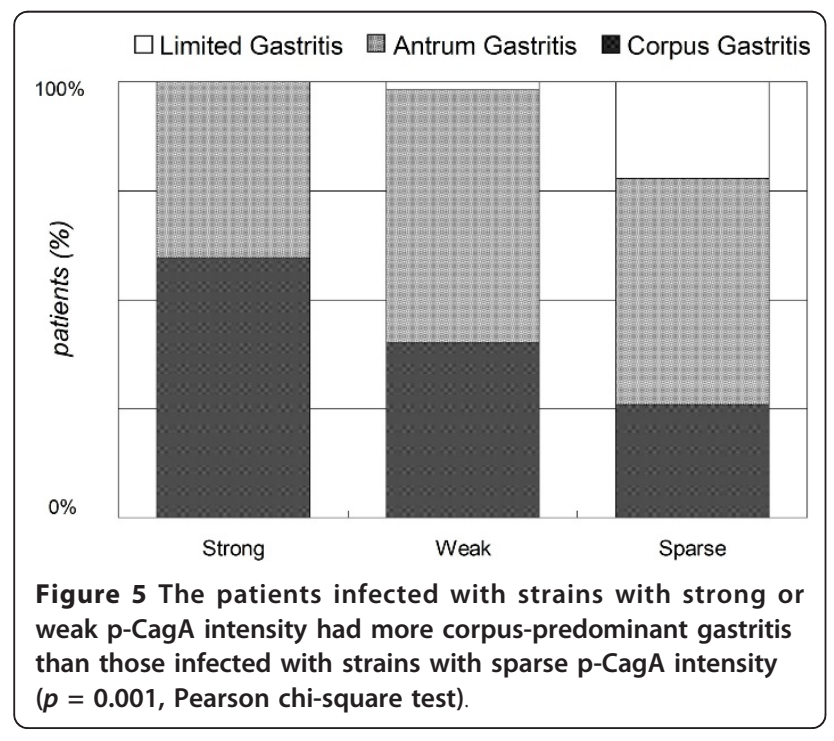

via CagA phosphorylation. However, there is no previous human study to evaluate the impact of the pCagA intensity on gastric histological changes. Thus, this study is first time to disclose that strains isolated from gastric cancer and IM patients had a stronger pCagA function as compared with strain from gastritis without IM patients (Figure 2). However, those were not significantly stronger than the strains from gastric or duodenal ulcer. This result can be explained that the IM and non-IM were both included into the gastric and duodenal ulcer subgroups to dilute the significance. This explanation may be also supported by a study showing that the intensities of p-CagA were not significantly different among different clinical diseases [22].

Moreover, as shown in Figure 3, the isolates from patients with cancer risk (i.e, patients with IM or cancer) had significantly stronger p-CagA intensity than those from patients without cancer risk $(p<0.001)$. This data further support that strong p-CagA increase the risk of developing gastric carcinogenesis from $H$. pylori infection. Furthermore, the patients with IM or cancer had severer acute and chronic inflammation in gastric histology. Also shown in Figure 4, the patients infected with stronger p-CagA $H$. pylori strains could correlate with severer acute or chronic gastritis $(p<$ $0.05)$. This indicated that the p-CagA intensity is closely 
related to provoke gastric inflammation in both patients with and without gastric cancers.

It is well known that the $H$. pylori-infected host has an increased risk of developing gastric cancer, once the gastric histology reveals a corpus-predominant pattern or a precancerous change such as IM [30-33]. We, therefore, further validated whether the infection of patients with strong p-CagA H. pylori strains is associated with an increased risk of such histological changes. As shown in Figure 5, strains with stronger p-CagA caused more often corpus-predominant gastritis $(p=0.001)$. Also shown in Figure 2, the strains isolated from patients of gastritis with IM had a significantly stronger p-CagA than those from gastritis patients without IM ( $p=$ 0.002). These data supported the hypothesis that the pCagA intensity of $H$. pylori isolates is closely related with the presence of IM.

In this study, instead of using all 469 stored strains, we systemically sampled 146 strains from our $H$. pylori database for the analysis of the p-CagA intensity. Both crude and adjusted odds ratio of the p-CagA intensity on IM were computed by logistical regression for the possible confounding factors, such as age, gender, and clinical disease. As shown in Table 2, the older age, female and stronger $\mathrm{p}$-CagA had higher risk of having IM. In the multivariable regression, patients infected with $H$. pylori strains with strong and weak p-CagA had a 10.45 and 3.93 times higher risk of having IM than those infected with strains with sparse p-CagA intensity.

The study is noteworthy in showing that, in a $100 \%$ cagA-genopositive area, the p-CagA intensity could be an important independent factor closely associated with an increased risk of precancerous changes such as IM. However, the assessment of the p-CagA intensity in $H$. pylori isolates may not be widely available for clinical application. Accordingly, it is worth conducting future studies to determine biomarkers to indirectly evaluate the p-CagA intensity of the infected host. Once a biomarker is available, it will be helpful to identify patients infected with $H$. pylori strains with stronger $\mathrm{p}$-CagA intensity, to determine the risk of gastric carcinogenesis in non-cancer patients, and then select these patients for earlier treatment.

\section{Conclusions}

In conclusion, patients infected with a $H$. pylori strain with stronger CagA phosphorylation ability have more severe chronic gastric inflammation with an increased risk to have corpus-predominant gastritis, gastric intestinal metaplasia, and cancer.

\section{List of abbreviations used}

p-CagA: phosphorylated-CagA; IM: intestinal metaplasia; cag-PAl: cag pathogenicity island; HPD: H. pylori density; AIS: acute inflammation score; CIS: chronic inflammation score; H. pylori: Helicobacter pylori
Acknowledgements

Financial support: This work was supported by grants from the National Scientific Council (NSC982314B006036), the Department of Health (DOH99TD-C-111-003), and the National Health Research Institute (NHRI-EX999908BI), Taiwan

\section{Author details}

${ }^{1}$ Department of Internal Medicine, Medical College, National Cheng Kung University, Sheng-Li Road, Tainan, Taiwan. ${ }^{2}$ Department of Pathology, Medical College, National Cheng Kung University, Sheng-Li Road, Tainan, Taiwan. ${ }^{3}$ Institute of Basic Medical Sciences, Medical College, National Cheng Kung University, Sheng-Li Road, Tainan, Taiwan. ${ }^{4}$ Institute of Clinical Medicine, Medical College, National Cheng Kung University, Sheng-Li Road, Tainan, Taiwan.

\section{Authors' contributions}

Guarantor of the article: Bor-Shyang Sheu, MD

Specific author contributions: Dr. CCH and SBS initiated and coordinated the study conduction. CHC and CWL enrolled the patients. YHB reviewed the gastric histology. $\mathrm{HKH}, \mathrm{SSM}$, and WJJ assessed the cagA genotype and $\mathrm{p}$ CagA intensity. All authors read and approved the final manuscript.

\section{Authors' information}

Chiao-Hsiung Chuang, MD: Institute of Clinical Medicine, Department of Internal Medicine, Medical College, National Cheng Kung University, Tainan, Taiwan.

Hsiao-Bai Yang, MD: Department of Pathology, Medical College, National Cheng Kung University, Tainan; Department of Pathology, Ton-Yen General Hospital, Hsinchu, Taiwan.

Shew-Meei Sheu, PhD: Institute of Basic Medical Sciences, Medical College, National Cheng Kung University, Tainan, Taiwan.

Kuei-Hsiang Hung, PhD: Institute of Basic Medical Sciences, Medical College, National Cheng Kung University, Tainan, Taiwan.

Jiunn-Jong Wu, PhD: Institute of Basic Medical Sciences, Medical College, National Cheng Kung University, Tainan, Taiwan.

Hsiu-Chi Cheng, MD, PhD: Institute of Clinical Medicine, Department of Internal Medicine, Medical College, National Cheng Kung University, Tainan, Taiwan.

Wei-Lun Chang, MD: Institute of Clinical Medicine, Department of Internal Medicine, Medical College, National Cheng Kung University, Tainan, Taiwan. Bor-Shyang Sheu, MD: Department of Internal Medicine, Institute of Clinical Medicine, Institute of Basic Medical Sciences, Medical College, National Cheng Kung University, Tainan, Taiwan.

Received: 27 November 2010 Accepted: 27 May 2011 Published: 27 May 2011

\section{References}

1. Suriani R, Colozza M, Cardesi E, Mazzucco D, Marino M, Grosso S, Sanseverinati S, Venturini I, Borghi A, Zeneroli ML: CagA and VacA Helicobacter pylori antibodies in gastric cancer. Can J Gastroenterol 2008, 22:255-258.

2. Wada Y, Ito M, Takata S, Tanaka S, Yoshihara M, Chayama K: Relationship between Helicobacter pylori tyrosine-phosphorylated CagA-related markers and the development of diffuse-type gastric cancers: a casecontrol study. Digestion 2010, 82:10-17.

3. Martin Guerrero JM, Hergueta Delgado P, Esteban Carretero J, Romero Castro R, Pellicer Bautista FJ, Herrerias Gutierrez JM: Clinical relevance of Helicobacter pylori CagA-positive strains: gastroduodenal peptic lesions marker. Rev Esp Enferm Dig 2000, 92:160-173.

4. Salehi Z, Jelodar MH, Rassa M, Ahaki M, Mollasalehi H, Mashayekhi F: Helicobacter pylori cagA status and peptic ulcer disease in Iran. Dig Dis Sci 2009, 54:608-613.

5. Hatakeyama M, Higashi H: Helicobacter pylori CagA: a new paradigm for bacterial carcinogenesis. Cancer Sci 2005, 96:835-843.

6. Cendron L, Couturier M, Angelini A, Barison N, Stein M, Zanotti G: The Helicobacter pylori CagD (HP0545, Cag24) protein is essential for CagA translocation and maximal induction of interleukin-8 secretion. J Mol Biol 2009, 386:204-217.

7. Lee IO, Kim JH, Choi YJ, Pillinger MH, Kim SY, Blaser MJ, Lee YC: Helicobacter pylori CagA phosphorylation status determines the gp130- 
activated SHP2/ERK and JAK/STAT signal transduction pathways in gastric epithelial cells. J Biol Chem 2010, 285:16042-1650.

8. Argent RH, Kidd M, Owen RJ, Thomas RJ, Limb MC, Atherton JC: Determinants and consequences of different levels of CagA phosphorylation for clinical isolates of Helicobacter pylori. Gastroenterology 2004, 127:514-523.

9. Wiedemann T, Loell E, Mueller S, Stoeckelhuber M, Stolte M, Haas R, Rieder G: Helicobacter pylori cag-Pathogenicity island-dependent early immunological response triggers later precancerous gastric changes in Mongolian gerbils. PLoS One 2009, 4:e4754.

10. Rieder G, Merchant JL, Haas R: Helicobacter pylori cag-type IV secretion system facilitates corpus colonization to induce precancerous conditions in Mongolian gerbils. Gastroenterology 2005, 128:1229-1242.

11. Torres LE, Melian K, Moreno A, Alonso J, Sabatier CA, Hernandez M, Bermudez L, Rodriguez BL: Prevalence of vacA, cagA and babA2 genes in Cuban Helicobacter pylori isolates. World J Gastroenterol 2009, 15:204-210.

12. Paniagua GL, Monroy E, Rodriguez R, Arroniz S, Rodriguez C, Cortes JL, Camacho A, Negrete E, Vaca S: Frequency of vacA, cagA and babA2 virulence markers in Helicobacter pylori strains isolated from Mexican patients with chronic gastritis. Ann Clin Microbiol Antimicrob 2009, 8:14.

13. Sheu BS, Yang HB, Yeh YC, Wu JJ: Helicobacter pylori colonization of the human gastric epithelium: a bug's first step is a novel target for us. $J$ Gastroenterol Hepatol 2010, 25:26-32.

14. Sheu BS, Sheu SM, Yang HB, Huang AH, Wu JJ: Host gastric Lewis expression determines the bacterial density of Helicobacter pylori in babA2 genopositive infection. Gut 2003, 52:927-932.

15. Sheu BS, Odenbreit S, Hung KH, Liu CP, Sheu SM, Yang HB, Wu JJ: Interaction between host gastric Sialyl-Lewis $X$ and $H$. pylori SabA enhances $\mathrm{H}$. pylori density in patients lacking gastric Lewis B antigen. Am J Gastroenterol 2006, 101:36-44.

16. Lai YP, Yang JC, Lin TZ, Wang JT, Lin JT: CagA tyrosine phosphorylation in gastric epithelial cells caused by Helicobacter pylori in patients with gastric adenocarcinoma. Helicobacter 2003, 8:235-243.

17. Argent RH, Hale JL, El-Omar EM, Atherton JC: Differences in Helicobacter pylori CagA tyrosine phosphorylation motif patterns between western and East Asian strains, and influences on interleukin-8 secretion. J Med Microbiol 2008, 57:1062-1067.

18. Jones KR, Joo YM, Jang S, Yoo YJ, Lee HS, Chung IS, Olsen CH, Whitmire JM, Merrell DS, Cha JH: Polymorphism in the CagA EPIYA motif impacts development of gastric cancer. J Clin Microbiol 2009, 47:959-968

19. Sheu SM, Sheu BS, Yang HB, Li C, Chu TC, Wu JJ: Presence of iceA1 but not cagA, cagC, cagE, cagF, cagN, cagT, or orf13 genes of Helicobacter pylori is associated with more severe gastric inflammation in Taiwanese. J Formos Med Assoc 2002, 101:18-23.

20. Yeh YC, Cheng HC, Chang WL, Yang HB, Sheu BS: Matrix metalloproteinase-3 promoter polymorphisms but not dupA-H. pylori correlate to duodenal ulcers in $\mathrm{H}$. pylori-infected females. BMC Microbiol 2010, 10:218.

21. Chuang CH, Sheu BS, Yang HB, Lee SC, Kao AW, Cheng HC, Chang WL, Yao WJ: Gender difference of circulating ghrelin and leptin concentrations in chronic Helicobacter pylori infection. Helicobacter 2009, 14:54-60.

22. Atherton JC, Blaser MJ: Coadaptation of Helicobacter pylori and humans: ancient history, modern implications. J Clin Invest 2009, 119:2475-2487.

23. Naito M, Yamazaki T, Tsutsumi R, Higashi H, Onoe K, Yamazaki S, Azuma T, Hatakeyama M: Influence of EPIYA-repeat polymorphism on the phosphorylation-dependent biological activity of Helicobacter pylori CagA. Gastroenterology 2006, 130:1181-1190.

24. Schmidt HM, Andres S, Nilsson C, Kovach Z, Kaakoush NO, Engstrand L, Goh KL, Fock KM, Forman D, Mitchell H: The cag PAl is intact and functional but HP0521 varies significantly in Helicobacter pylori isolates from Malaysia and Singapore. Eur J Clin Microbiol Infect Dis 2010, 29:439-451.

25. Backert S, Churin Y, Meyer TF: Helicobacter pylori type IV secretion, host cell signalling and vaccine development. Keio J Med 2002, 51(Suppl 2):6-14.

26. Acosta N, Quiroga A, Delgado P, Bravo MM, Jaramillo C: Helicobacter pylori CagA protein polymorphisms and their lack of association with pathogenesis. World J Gastroenterol 2010, 16:3936-3943.

27. Uchida T, Nguyen LT, Takayama A, Okimoto T, Kodama M, Murakami K, Matsuhisa T, Trinh TD, Ta L, Ho DQ, et al: Analysis of virulence factors of
Helicobacter pylori isolated from a Vietnamese population. BMC Microbiol 2009, 9:175.

28. Shibata W, Hirata Y, Maeda S, Ogura K, Ohmae T, Yanai A, Mitsuno Y, Yamaji Y, Okamoto M, Yoshida H, et al: CagA protein secreted by the intact type IV secretion system leads to gastric epithelial inflammation in the Mongolian gerbil model. J Pathol 2006, 210:306-314.

29. Batista SA, Rocha GA, Rocha AM, Saraiva IE, Cabral MM, Oliveira RC, Queiroz DM: Higher number of Helicobacter pylori CagA EPIYA C phosphorylation sites increases the risk of gastric cancer, but not duodenal ulcer. BMC Microbiol 2011, 11:61.

30. Uemura N, Okamoto S, Yamamoto S, Matsumura N, Yamaguchi S, Yamakido M, Taniyama K, Sasaki N, Schlemper RJ: Helicobacter pylori infection and the development of gastric cancer. N Engl J Med 2001, 345:784-789.

31. Hung KH, Wu JJ, Yang HB, Su LJ, Sheu BS: Host Wnt/beta-catenin pathway triggered by Helicobacter pylori correlates with regression of gastric intestinal metaplasia after H. pylori eradication. J Med Microbiol 2009, 58:567-576.

32. Sheu BS, Yang HB, Sheu SM, Huang AH, Wu JJ: Higher gastric cycloxygenase-2 expression and precancerous change in Helicobacter pylori-infected relatives of gastric cancer patients. Clin Cancer Res 2003, 9:5245-5251.

33. Polk DB, Peek RM Jr: Helicobacter pylori: gastric cancer and beyond. Nat Rev Cancer 2010, 10:403-414.

doi:10.1186/1471-2180-11-121

Cite this article as: Chuang et al:: Helicobacter pylori with stronger intensity of CagA phosphorylation lead to an increased risk of gastric intestinal metaplasia and cancer. BMC Microbiology 2011 11:121.

\section{Submit your next manuscript to BioMed Central and take full advantage of:}

- Convenient online submission

- Thorough peer review

- No space constraints or color figure charges

- Immediate publication on acceptance

- Inclusion in PubMed, CAS, Scopus and Google Scholar

- Research which is freely available for redistribution

Submit your manuscript at www.biomedcentral.com/submit
C Biomed Central 